\title{
Investigational Product Retest and Expiry Document
}

National Cancer Institute

\section{Source}

National Cancer Institute. Investigational Product Retest and Expiry Document. NCI

Thesaurus. Code C125435.

A document describing the batch retesting and analyses of the investigational product (IP). 